\title{
Supersymmetric models with broken Lorentz invariance.
}

\author{
Arthur Marakulin ${ }^{1, \star}$ and Sergey Sibiryakov ${ }^{1,2,3, \star \star}$ \\ ${ }^{1}$ Institute for Nuclear Research of the Russian Academy of Sciences, Moscow, Russia \\ ${ }^{2}$ CERN Theory division, Geneva, Switzerland; \\ ${ }^{3}$ Institut de Physique, EPFL, Lausanne, Switzerland
}

\begin{abstract}
Several supersymmetric theories with broken Lorentz invariance are considered. We study at the component level Lorentz violating representations of the supersymmetry algebra and construct Lagrangians for the scalar and vector supermultiplets with broken Lorentz invariance. Lorentz violating model for the gravitational supermultiplet is constructed using the superfield formalism as supersymmetric extension of the linearized Einstein-aether theory. The most general Lagrangian of the linearized Einstein-aether supergravity is constructed. We show that the Lagrangian for this model is unique and obtain its bosonic part in components. The constraints imposed by supersymmetry on the parameters of the theory are obtained. The phenomenological consequences of the model are discussed.
\end{abstract}

\section{Introduction}

Incompatibility of the principles of quantum field theory with general relativity is one of the most important problems in modern theoretical physics. A potential way out of this situation consists in restricting the domain of validity of some basic postulates of general relativity and abandoning them at high energy scales.

There is a number of motivations for abandoning Lorentz invariance at small scales. The first motivation to study models with Lorentz breaking is their rich phenomenology[1]. Another motivation comes from the theory. A number of Lorentz violating models of gravity demonstrate better quantum behavior than general relativity. A promising approach to quantization of gravity based on abandoning the Lorentz invariance has been proposed by Hořava [2] and extended by several authors [3, 4]. Hořava gravity is closely related [5, 6] to the so-called Einstein-aether model [1]. In the latter model violation of the Lorentz invariance is described by the time-like vector field $u^{m}$ with unit norm $\left(u^{m} u_{m}=-1\right)$ called aether that minimally couples to the Einstein-Hilbert action for gravity,

$$
S=S_{G R}-\frac{1}{2} \int d^{4} x \sqrt{-g}\left(c_{1}\left(\nabla_{n} u_{m}\right)^{2}+c_{2}\left(\nabla_{m} u_{m}\right)^{2}+c_{3} \nabla_{n} u_{m} \nabla^{m} u^{n}-c_{4} u^{r} u^{s} \nabla_{r} u_{m} \nabla_{s} u^{m}\right) .
$$

The theory contains four dimensionless parameters $c_{i}, i=1,2,3,4$. In general case of the theory there are strong constraints on these parameters, because the PPN-parameters depends on the constants in the Einsten-aether model [1]

\footnotetext{
${ }^{\star}$ e-mail: marakulin@physics.msu.ru

$\star \star$ e-mail: sergey.sibiryakov@cern.ch
} 


$$
\begin{gathered}
\alpha_{1}=-8 \frac{c_{3}^{2}+c_{1} c_{4}}{2 c_{1}-c_{1}^{2}+c_{3}^{2}}, \\
\alpha_{2}=\frac{\alpha_{1}}{2}-\frac{\left(c_{1}+2 c_{3}-c_{4}\right)\left(2 c_{1}+3 c_{2}+c_{3}+c_{4}\right)}{\left(c_{1}+c_{2}+c_{3}\right)\left(2-c_{1}-c_{4}\right)},
\end{gathered}
$$

and these are constrained from observations in the Solar System [7]:

$$
\left|\alpha_{1}\right| \lesssim 10^{-4} ;\left|\alpha_{2}\right| \lesssim 4 \times 10^{-7}
$$

A phenomenologically acceptable theory with Lorentz violation in the ultraviolet must incorporate a mechanism that ensures emergence of Lorentz invariance at low energies. One of the possible mechanisms is based on non-relativistic supersymmetry [8,9]. As remarked, supersymmetry does not necessarily require Lorentz invariance. SUSY enforces emergence of Lorentz symmetry at low energies in the Standard Model, even if the high-energy theory is not Lorentz invariant. It is the natural motivation to consider supersymmetric models with Lorentz breaking for better theoretical understanding of their structure.

The main purpose of the present work is to consider the existence and uniqueness of the supersymmetric Lorentz violating field theories. We study at the component level Lorentz violating representations of the supersymmetry algebra and construct Lagrangians for the scalar and vector supermultiplets with broken Lorentz invariance. Lorentz violating model for the gravitational supermultiplet is constructed using the superfield formalism as supersymmetric extension of the linearized Einstein-aether theory. We consider the coupling of supersymmetric aether to supergravity. We use the superfield formulation of the linearized non-minimal supergravity to construct a Lagrangian for the aether field coupled to linearized gravity and prove that it is unique, up to the choice of a single parameter.

\section{Supersymmetry and Lorentz breaking}

Apart from the paper[9], we start from the on-shell field theories in components. Let's consider massless supermultiplet $\left(A, \Psi^{\alpha}\right)$ consisting of the scalar and spinor fields. To analyze the existence and unity of the scalar supermultiplet theory with broken Lorentz invariance we consider the relevant representation for the supersymmetry algebra with Lorentz invariance broken by the timelike vector $u_{m}$ with unit norm. It is possible to obtain after straightforward calculations, that supersymmetry algebra closen on-shell as follows: $\left[\delta_{\xi} \delta_{\eta}\right] X=2 i\left(\eta \sigma_{m} \bar{\xi}-\xi \sigma_{m} \bar{\eta}\right) \partial_{m} X$, takes the form

$$
\left\{\begin{array}{l}
\delta_{\xi} A=\xi \Psi \\
\delta_{\xi} \Psi_{\alpha}=2 i\left(\sigma_{m} \bar{\xi}\right)_{\alpha} \partial_{m} A+C_{1} i u_{m} \xi_{\alpha} \partial_{m} \bar{A}+C_{2} i u_{m}\left(\sigma_{m n} \xi\right)_{\alpha} \partial_{n} \bar{A}
\end{array}\right.
$$

It is the most general form of the supersymmetric transformations in the relevant case. The requirement to the theory to be set by Lagrangian, consistent with the equations of motion and invariant under the supersymmetric transformations, leads us to the constraints

$$
C_{1}=C_{2}=0,
$$

and to the consequence that there is no non-trivial Lorentz violating supersymmetric model containing the most simple set of fields. 
Thus, there is no any new possibilities for the constructing the supersymmetic model with scalar and spinor without Lorentz invariance for the scalar supermultiplet. Another analysis is required if we consider several scalar and several spinor fields $\left(A^{i}, \Psi_{\alpha}^{i}\right)$. In this case all of the coefficients will have two indices $\left(C^{i j}\right)$, and the transformations from the supersymmetry algebra closed on-shell will be matrices.

$$
\left\{\begin{array}{l}
\delta_{\xi} A^{i}=\xi \Psi^{i} \\
\delta_{\xi} \Psi_{\alpha}^{i}=2 i\left(\sigma_{m} \bar{\xi}\right)_{\alpha} \partial_{m} A^{i}+C_{1}^{i j} i u_{m} \xi_{\alpha} \partial_{m} \bar{A}^{j}+C_{2}^{i j} i u_{m}\left(\sigma_{m n} \xi\right)_{\alpha} \partial_{n} \bar{A}^{j}
\end{array}\right.
$$

The requirement of the Lagrangian existence leads us to the constraints

$$
\begin{gathered}
C_{1}^{i j}=-C_{1}^{j i}, \\
C_{2}^{i j}=0,
\end{gathered}
$$

and to the non-trivial supersymmetric model with Lorentz breaking, containing a set of spinor and scalar fields

$$
L=i \Psi^{i} \sigma_{m} \partial_{m} \bar{\Psi}^{i}+\bar{A}^{i} \square A^{i}-\frac{i}{4} C_{1}^{i j} u_{m}\left(\Psi^{i} \partial_{m} \Psi^{j}+\text { h. c. }\right)-\frac{1}{2}\left(C_{1}^{2}\right)^{i j} u_{m} u_{n} \partial_{m} A^{i} \partial_{n} \bar{A}^{j}
$$

Thus, existence of Lorentz violating models for the scalar supermultiplets depends on the number of scalars and spinors. This fact is important for the case of one scalar and one spinor, because the model in this case can be only trivial.

By the analogical calculations one can obtain there is no non-trivial Lorentz-violating supersymmetric models for the vector supermultiplet $\left(v_{m}, \lambda_{\alpha}\right)$. The existence of the models for the scalar supermultiplet depends on the number of scalars and spinors, and also on the fact if we require the existence of the Lagrangian of the theory. These results agree with the superfield formalism consequences[9], and may indirectly confirm the correctness of the superfield formalism in the Lorentz violating case.

The next natural question is if non-trivial theories for the gravitational multiplet exist. The first step in answering this question was made in [8]. The authors of the paper cosidered the supersymmetric extension of the aether model in terms of chiral vector superfiels:

$$
U^{m}=u^{m}\left(x_{L}\right)+\sqrt{2} \theta \eta^{m}\left(x_{L}\right)+\theta^{2} G^{m}\left(x_{L}\right)
$$

where $\left(x_{L}^{m}=x^{m}+i \theta \sigma^{m} \bar{\theta}, \theta^{\alpha}\right)$. The unit norm constraint on the super-aether is similar to the ordinary case:

$$
U_{m} U^{m}=-1
$$

The authors showed that the most general super-aether action

$$
S=\int d^{8} z f\left(U_{m} \bar{U}^{m}\right)+\int d^{6} z \Lambda\left(U_{m} U^{m}+1\right)
$$

leads to the constraints:

$$
\begin{gathered}
c_{2}+c_{3}=0, \\
c_{4}=0 .
\end{gathered}
$$


It has been also shown that in the supersymmetric extension of the Einstein-aether theory based on chiral vector super-aether field interactions between the aether and the fields of the Standard Model are suppressed.

This cannot be realized within the minimal $N=1$ supergravity which does not allow to define a chiral aether vector superfield $\left(\bar{\nabla}_{\dot{\alpha}} U^{a}=0\right)$ : anti-commutator of two supercovariant derivatives with spinor indices acting on a vector superfield does not vanish prohibiting to impose the chirality condition. This problem is resolved in the non-minimal formulation of the $N=1$ supergravity.

\section{Supergravity with broken Lorentz invariance}

Non-minimal linearized supergravity is formulated [10] in terms of real superfield $H^{m}$ and so-called linear compensator $\Gamma\left(\bar{D}^{2} \Gamma=0\right)$ as a gauge theory with the action

$$
\begin{aligned}
S_{S G}= & \frac{1}{\varkappa^{2}} \int d^{8} z\left[\frac{1}{4}\left(\left(\partial_{k} H_{m}\right)^{2}-\left(\Delta_{k} H_{m}\right)^{2}\right)+\frac{n+1}{2 n}\left(\partial_{m} H^{m}\right)^{2}+\frac{n+1}{2}\left(\Delta_{m} H^{m}\right)^{2}-\right. \\
& \left.-i \frac{3 n+1}{2 n} \partial_{m} H^{m}(\Gamma-\bar{\Gamma})+\frac{3 n+1}{2} \Delta_{m} H^{m}(\Gamma+\bar{\Gamma})+\frac{9 n^{2}-1}{8 n}\left(\Gamma^{2}+\bar{\Gamma}^{2}\right)+\frac{(3 n+1)^{2}}{4 n} \Gamma \bar{\Gamma}\right] .
\end{aligned}
$$

where $\Delta_{k} H_{m}=\frac{1}{4} \bar{\sigma}_{k}^{\dot{\alpha} \alpha}\left[\bar{D}_{\dot{\alpha}}, D_{\alpha}\right] H_{m}$. The action is invariant under super-gauge transformations

$$
\begin{gathered}
\delta H_{\alpha \dot{\alpha}}=\bar{D}_{\dot{\alpha}} L_{\alpha}-D_{\alpha} \bar{L}_{\dot{\alpha}} \\
\delta \Gamma=-\frac{n+1}{4(3 n+1)} \bar{D}^{2} D^{\alpha} L_{\alpha}+\frac{1}{4} \bar{D}^{\dot{\alpha}} D^{2} \bar{L}_{\dot{\alpha}}
\end{gathered}
$$

We construct linearized Einstein-aether supergravity theory in terms the latter superfields and the chiral vector super-aether field which is considered as consisting of the real vacuum and small perturbation:

$$
U^{a}=w^{a}+V^{a}
$$

The super-aether field gauge transformation

$$
\delta V^{a}=w^{b} M_{b}^{a}
$$

where

$$
M_{a b}=\frac{1}{4}\left(\sigma_{a b}\right)_{\beta}{ }^{\alpha} D_{\alpha} \bar{D}^{2} L^{\beta}+\frac{1}{4}\left(\bar{\sigma}_{a b}\right)_{\dot{\beta}}^{\dot{\alpha}} \bar{D}^{\dot{\beta}} D^{2} \bar{L}_{\dot{\alpha}}
$$

is consistent with the chirality condition,

$$
\bar{D}_{\dot{\alpha}} V^{c}=-w^{b} \Phi_{\dot{\alpha} b}^{c} .
$$

where

$$
\Phi_{\dot{\alpha} b c}=-\frac{1}{4}\left(\sigma_{b c}\right)_{\alpha}{ }^{\beta} \bar{D}^{2} D^{\alpha} H_{\beta \dot{\alpha}}-\left(\bar{\sigma}_{b c}\right)^{\dot{\beta}} \bar{D}_{\dot{\alpha}} \Gamma
$$

is superconnection.

To carry out the Lagrangian of the theory one have to write down supersymmetric transformations of all possible independent terms quadratically dependent on the aether or gravitational superfields. 
Coefficients in these transformations define the system of linear equation to be solved. A straightforward but tedious calculation yields [11] the most general and unique Lagrangian invariant under gauge transformations with one free parameter $C$ :

$$
\begin{aligned}
& S_{\mathfrak{x}}=\frac{C}{2 \kappa^{2}} \int d^{8} z\left[V_{a} \bar{V}^{a}+i w^{a} w^{b} \partial_{a} H_{b}(\Gamma-\bar{\Gamma})+w^{a} w^{b} \Delta_{a} H_{b}(\Gamma+\bar{\Gamma})+\right. \\
&+\frac{1}{4}\left(\Delta_{k} H_{m} \Delta^{k} H^{m}-\partial_{k} H_{m} \partial^{k} H^{m}-\left(\Delta_{m} H^{m}\right)^{2}+\left(\partial_{m} H^{m}\right)^{2}\right)+ \\
&\left.\quad+\frac{i}{4} \partial_{m} H^{m}(\Gamma-\bar{\Gamma})+\frac{1}{4} \Delta_{m} H^{m}(\Gamma+\bar{\Gamma})+\frac{3}{8}\left(\Gamma^{2}+\bar{\Gamma}^{2}\right)\right],
\end{aligned}
$$

\section{From superfields to component fields}

In the real world supersymmetry must be broken. As shown in [8], a soft breaking of supersymmetry gives mass to the imaginary component of the super-aether, whereas its real part remains massless. At low energies, the theory is described by the action (1), where the parameters $c_{i}, i=1,2,3,4$, however, are constrained by supersymmetry. To find these constraints, we write the bosonic part of the Lagrangian (24) in terms of component fields. We use the bosonic parts of the gravitational superfield in the Wess-Zumino gauge [10] and use the local Lorentz transformations to make the tetrad symmetric $\left(e_{m n}=e_{n m}=\frac{1}{2} h_{m n}\right)$,

$$
\left.H_{m}(x, \theta, \bar{\theta})\right|_{\mathrm{bos}}=\frac{1}{2} \theta \sigma^{n} \bar{\theta} e_{m n}(x)+\theta^{2} \bar{\theta}^{2} d_{m}(x) ;
$$

We use the linear compensator in terms of the "left" coordinates $\left(x_{L}^{m}=x^{m}+i \theta \sigma^{m} \bar{\theta}, \theta^{\alpha}\right)$

$$
\left.\Gamma\left(x_{L}, \theta, \bar{\theta}\right)\right|_{\mathrm{bos}}=\frac{n+1}{2(3 n+1)} h\left(x_{L}\right)+\theta^{2} B\left(x_{L}\right)+\theta \sigma^{a} \bar{\theta}\left(v_{a}\left(x_{L}\right)+i \mu_{a}\left(x_{L}\right)\right) ;
$$

and the aether superfield

$$
\left.V_{b}\left(x_{L}, \theta, \bar{\theta}\right)\right|_{\text {bos }}=v_{b}\left(x_{L}\right)+\theta^{2} G_{b}\left(x_{L}\right)+\theta \sigma^{k} \bar{\theta} f_{b k}\left(x_{L}\right),
$$

where $v_{a}=v_{a}^{R}+i v_{a}^{I}$ is the first order aether perturbation, and

$$
f_{b k}=w_{c} d_{p} \operatorname{tr}\left(\sigma_{b c} \sigma_{p k}\right)+\frac{i}{2} w_{c} \partial_{m} h_{n p} \operatorname{tr}\left(\sigma_{k} \bar{\sigma}_{p} \sigma_{b c} \sigma_{n m}\right)+w_{c}\left(v_{p}+i \mu_{p}\right) \operatorname{tr}\left(\bar{\sigma}_{b c} \bar{\sigma}_{k p}\right) .
$$

Expanding in the Grassmann coordinates we obtain the bosonic part of the Lagrangian in components. Integrating out the auxiliary fields under the assumption $C \ll 1$ through order $O(\sqrt{C})$ we reduce to the linearized Lagrangian of the Einstein-aether supergravity through order $O(C)$

$$
\begin{aligned}
& L=\frac{1}{2 \varkappa^{2}}\left\{\frac{1}{4} h_{k m} \square h^{k m}+\frac{1}{2} \partial^{k} h_{k m} \partial_{l} h^{l m}-\frac{1}{2} \partial_{k} h^{k m} \partial_{m} h+\right. \\
&+\frac{1}{4} \partial_{m} h \partial^{m} h-\partial_{m} \hat{v}_{a}^{R} \partial^{m} \hat{v}^{R, a}-\partial_{m} \hat{v}_{a}^{I} \partial^{m} \hat{v}^{I, a}+\sqrt{C} \hat{v}^{R, a} w^{b}\left(\partial_{b} \partial^{k} h_{k a}-\partial_{a} \partial^{k} h_{k b}\right)- \\
& \quad-\frac{C}{4} w^{a} w^{b}\left(\partial_{a} h_{m n}-\partial_{m} h_{n a}\right)\left(\partial_{b} h^{m n}-\partial^{m} h_{b}^{n}\right)-\frac{C}{2} w^{a} w^{b} \partial_{a} \hat{v}^{I, m} \partial_{b} \hat{v}_{m}^{I}+ \\
&\left.+\frac{C}{2}\left(\partial_{a} \hat{v}^{I, a}\right)^{2}-C w^{b} w^{c} \epsilon_{b k a m} \partial^{k} \hat{v}^{R, a} \partial_{c} \hat{v}^{I, m}+O\left(C^{3 / 2}\right)\right\} .
\end{aligned}
$$

In this expression we have canonically normalized the aether perturbations, so their leading kinetic term became of order $1: v_{a}^{R, I} \mapsto \hat{v}_{a}^{R, I}=\sqrt{C} v_{a}^{R, I}[11]$. 


\section{Discussion}

Apart from the usual gravitational coupling, the theory is described by a single dimensionless constant $C$. When restricted to the case of real aether, $\hat{v}_{a}^{I}=0$, the Lagrangian (29) coincides with the quadratic part of the Einstein-aether Lagrangian 1 for the choice of couplings: $c_{1}=C, c_{2}=c_{3}=c_{4}=0$. Thus we conclude that SUSY reduces the number of free parameters in the Einstein-aether model from four down to one.

Modern experimental tests provide tight bounds on the parameters of Lorentz violation. In general, the Einstein-aether model gives rise to non-zero values of the post-Newtonian parameters $\alpha_{1,2}$ that can be expressed in terms of $c_{i}(2,3)$. In the supersymmetric model $c_{2,3,4}=0$ and

$$
\alpha_{1}=0, \quad \alpha_{2}=-\frac{2 C}{2-C},
$$

so the constraints translate into a bound on $C$ :

$$
C \lesssim 10^{-7} \text {. }
$$

More strong constraints to the parameter can be found from the pulsar astronomy[12]. This gives the bound,

$$
C<1.6 \times 10^{-9}
$$

but note that these observations are strictly relevant to the strong field case and should be used for the non-linear model. We'll briefly outline it at the end of the paper.

Another phenomenological consequence is the connection between structure of the supersymmetric algebra and the small perturbations velocity in the theory. It is curious that in supersymmetric theory of gravity the velocities of small perturbations is only luminal or superluminal[11]. In the Einstein-aether supergravity several modes with different helicities have the unit velocity, and for the others, including graviton,

$$
s^{2}=1+C
$$

where $s$ describes the linear dispersion relation in the theory:

$$
E=s \cdot p
$$

Due to the model properties [1] superluminal velocities don't lead to any disturbances in the theory. The fact that graviton velocity in the theory is over the speed of light can be used in the possible experimental tests of the model from the gravitational waves observations [13].

Recent extension of the model will be its generalization to the full non-linear supergravity case. It can be simply done in the superfield formalism, using notification of $[14,15]$, but the full analysis of the model and the reduction from theory in superspace to component fields are technically difficult and tedious. Nevertheless, this work will open the way to study possible manifestations of the model in cosmology, in particular, the effects of the additional fermionic and bosonic fields present in the model on the dynamics of the early universe. Other possible extensions of the model are the study of the different helicity mode velocities in different supersymmetric models with Lorentz breaking and its connection to the superalgebra structure, the effects of spontaneous supersymmetry breaking in the theory, etc. We leave it to the future works.

The work has been supported by the Russian Science Foundation grant 14-22-00161. 


\section{References}

[1] T. Jacobson, PoS QG-PH, 020 (2007).

[2] P. Horava, Physical Review D, 79, 8, 084008 (2009).

[3] D. Blas, O. Pujolas, O., and S. Sibiryakov, (2009). JHEP, 0910, 029 (2009)

[4] D. Blas, O. Pujolas, O., and S. Sibiryakov, Phys. Rev. Lett. 104, 181302, (2010)

[5] T. Jacobson, Phys. Rev. D 81, 101502 (2010) Erratum: [Phys. Rev. D 82, 129901 (2010)], Phys. Rev. D 89, 081501 (2014)

[6] D. Blas, O. Pujolas and S. Sibiryakov, JHEP 1104, 018 (2011)

[7] B. Z. Foster and T. Jacobson. Physical Review D, 73, 6, 064015, 1-9 (2006).

[8] O. Pujolas and S. Sibiryakov, JHEP, 1201, 062 (2012).

[9] S. Groot Nibbelink and M. Pospelov, Phys. Rev. Lett. 94, 081601 (2005).

[10] I. L. Buchbinder and S. M. Kuzenko, Ideas and methods of supersymmetry and supergravity, or A walk through superspace. (Institute of Physics Pub., 1998).

[11] A. Marakulin and S. Sibiryakov, arXiv:1610.07805.

[12] K. Yagi, D. Blas, N. Yunes and E. Barausse, Phys. Rev. Lett. 112, no. 16, 161101 (2014) Phys. Rev. D 89, no. 8, 084067 (2014) Erratum: [Phys. Rev. D 90, no. 6, 069902 (2014)] Erratum: [Phys. Rev. D 90, no. 6, 069901 (2014)]

[13] J. Ellis, N. E. Mavromatos and D. V. Nanopoulos, Mod. Phys. Lett. A 31, 1650155 (2016)

[14] G. Girardi, R. Grimm, M. Muller and J. Wess, Z. Phys. C 26, 123 (1984).

[15] G. Girardi, M. Muller and J. Wess, Z. Phys. C 26, 427 (1984). 